Korean J. Math. 21 (2013), No. 3, pp. 223-236

http://dx.doi.org/10.11568/kjm.2013.21.3.223

\title{
SOME PROPERTIES OF SCHENSTED ALGORITHM USING VIENNOT'S GEOMETRIC INTERPRETATION
}

\author{
JAEJIN LEE
}

\begin{abstract}
Schensted algorithm was first described in 1938 by Robinson [5], in a paper dealing with an attempt to prove the correctness of the Littlewood-Richardson rule. Schensted [9] rediscovered Schensted algorithm independently in 1961 and Viennot [12] gave a geometric interpretation for Schensted algorithm in 1977. In this paper we describe some properties of Schensted algorithm using Viennot's geometric interpretation.
\end{abstract}

\section{Introduction}

Let $\lambda=\left(\lambda_{1}, \lambda_{2}, \ldots, \lambda_{l}\right)$ be a partition of the nonnegative integer $n$, denoted $\lambda \vdash n$, so $\lambda$ is a weakly decreasing sequence of positive integers summing to $n$. We will also let $\lambda$ stand for the Ferrers diagram $D_{\lambda}$ of $\lambda$ written in English notation with $\lambda_{i}$ nodes or cells in the $i$ th row from the top.

Given $\lambda \vdash n$, a standard Young tableau $T$ of shape $\lambda$ is a filling of the diagram $D_{\lambda}$ with positive integers $1,2, \ldots, n$ such that rows and columns strictly increase. For example,

$\begin{array}{lll}1 & 3 & 5 \\ 2 & 6 & \\ 4 & 7 & \end{array}$

Received July 4, 2013. Revised August 1, 2013. Accepted August 2, 2013. 2010 Mathematics Subject Classification: 05E10.

Key words and phrases: partition, standard Young tableau, $P$-tableau, $Q$-tableau, Schensted algorithm.

(c) The Kangwon-Kyungki Mathematical Society, 2013.

This is an Open Access article distributed under the terms of the Creative commons Attribution Non-Commercial License (http://creativecommons.org/licenses/by -nc/3.0/) which permits unrestricted non-commercial use, distribution and reproduction in any medium, provided the original work is properly cited. 
is a standard Young tableau of the shape $(3,2,2)$.

There is a remarkable combinatorial correspondence associated with the theory of symmetric functions, called the Schensted algorithm.

THEOREM 1.1. (Schensted algorithm) Let $S_{n}$ be the symmetric group of degree $n$. Then there is a bijection

$$
\pi \mapsto(P, Q)
$$

between permutations $\pi$ of $S_{n}$ and the set of all pairs $\left(P_{\lambda}, Q_{\lambda}\right)$ of standard Young tableaux of the same shape $\lambda$, where $\lambda \vdash n$.

It was first described in 1938 by Robinson [5], in a paper dealing with an attempt to prove the correctness of the Littlewood-Richardson rule. Schensted algorithm was rediscovered independently by Schensted [9] in 1961, whose main objective was counting permutations with given lengths of their longest increasing and decreasing subsequences. Schensted correspondence about increasing and decreasing subsequences is extended by C. Greene [2], to give a direct interpretation of the shape of the standard Young tableaux corresponding to a permutation. The combinatorial significance of Schensted algorithm was indicated by Schützenberger [11], who introduced the evacuation algorithm. Knuth [4] gave a generalization of the Schensted algoritm, where standard Young tableaux are replaced by column strict tableaux, and permutations are replaced by multi-permutations. And he described conditions for two permutation to have the same $P$-tableaux under Schensted algorithm. In [12] Viennot gave a geometric interpretation for Schensted algorithm.

After Knuth generalized Schensted algorithm to column strict tableaux, various analogs of the Schented algorithm came: versions for rim hook tableaux [10], shifted tableaux ([6]), oscillating tableaux [1], skew tableaux [8], and shifted rim hook tableaux [3].

The bijection in Theorem 1.1 is denoted $\pi \leftrightarrow(P, Q)$ or $\pi \stackrel{[\mathrm{S}]}{\longmapsto}$ $(P(\pi), Q(\pi))$ and $P(\pi), Q(\pi)$ are called the $P$-tableau and $Q$-tableau of $\pi$, respectively. For example, if $\pi=3 a 7 b 24 d 5$ e $916 c 8 \in S_{14}$, then the $P$-tableau and $Q$-tableau of $\pi$ are given as

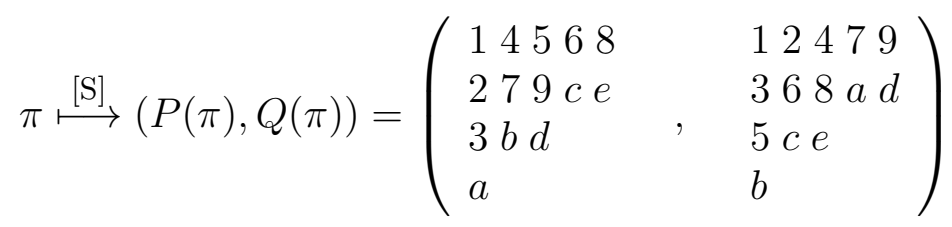


where $a=10, b=11, c=12, d=13$ and $e=14$.

In this paper we describe the ways to find $P$-tableaux and $Q$-tableaux of permutations $\pi^{r}, \pi^{*}$ and $\pi^{\#}$ without using Schützenberger's evacuation algorithm.

Section 2 gives Viennot's geometric interpretation for Schensted algorithm. In Section 3 we describe the ways to find $P$-tableaux and $Q$-tableaux of permutations $\pi^{r}, \pi^{*}$ and $\pi^{\#}$ using Viennot's geometric interpretation for Schensted algorithm.

\section{Geometric interpretation for Schensted algorithm}

In this section we describe Viennot's geometric interpretation for Schensted algorithm. See [12] or [7] for further exposition.

EXAMPLE 2.1. Let

$$
\pi=4236517 \in S_{7}
$$

- Consider the first quadrant of the Cartesian plane. Given a permutation $\pi=x_{1} x_{2} \cdots x_{n}$, represent $x_{i}$ by a box with coordinates $\left(i, x_{i}\right)$. See the figure 1 .

- Imagine a light shining from the origin so that each box casts a shadow with boundaries parallel to the coordinate axes. The shadow cast by the box at $(4,6)$ looks like the figure 2 .

- Consider those points of the permutation that are in the shadow of no other point. In this case $(1,4),(2,2)$, and $(6,1)$. The first shadow line, $L_{1}$, is the boundary of the combined shadows of these boxes. In the figure 3, the appropriate line has been thickened. Note that this is a broken line consisting of line segments and exactly one horizontal and one vertical ray. To form the second shadow line, $L_{2}$, one removes the boxes on the first shadow line and repeats this procedure.

Given a permutation displayed in the plane, we form its shadow lines $L_{1}, L_{2}, \ldots$ as follows. Assuming that $L_{1}, \ldots, L_{i-1}$ have been constructed, remove all boxes on these lines. Then $L_{i}$ is the boundary of the shadow of the remaining boxes. The $x$-coordinate of $L_{i}$ is

$x_{L_{i}}=$ the $x$-coordinate of $L_{i}$ 's vertical ray

and the $y$-coordinate is

$y_{L_{i}}=$ the $y$-coordinate of $L_{i}$ 's horizontal ray

The shadow lines make up the shadow diagram of $\pi$. 


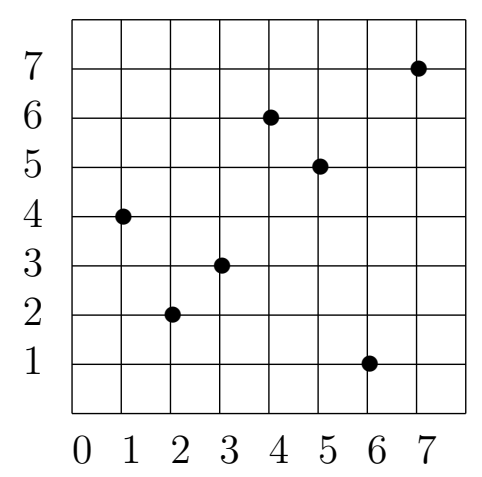

Figure 1 . The coordinates $\left(i, x_{i}\right)$ of $\pi$.

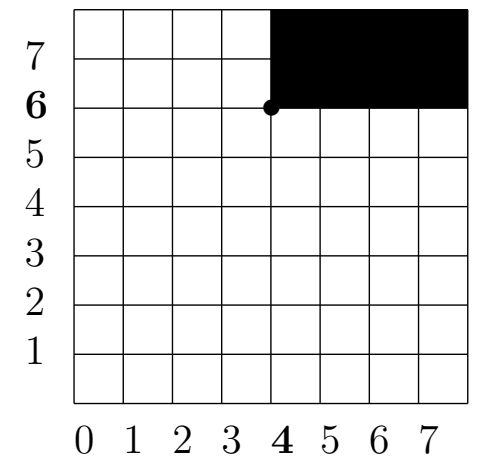

Figure 2. The shadow at $(4,6)$.

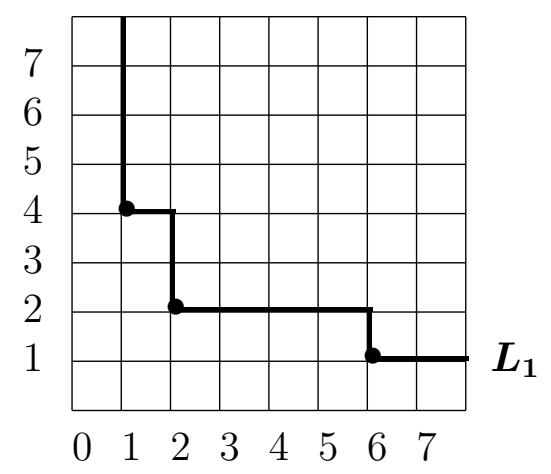

Figure 3 . The first shadow line, $L_{1}$. 
EXAMPLE 2.2. In the previous example, there are four shadow lines, and their $x$ - and $y$-coordinates are shown above and to the left of the figure 4 , respectively.

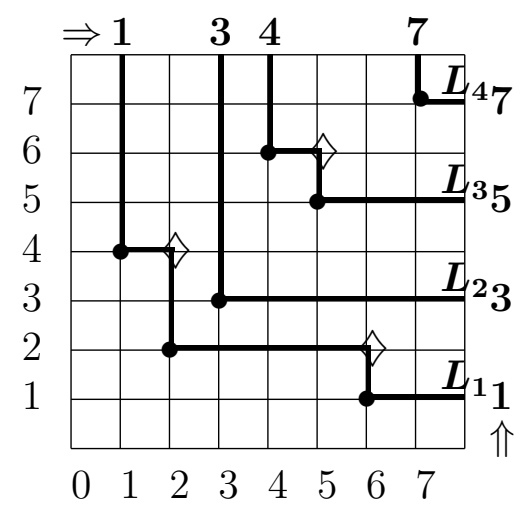

Figure 4. Four shadow lines for $\pi=4236517$

Compare the coordinates of our shadow lines with the first rows of the tableaux

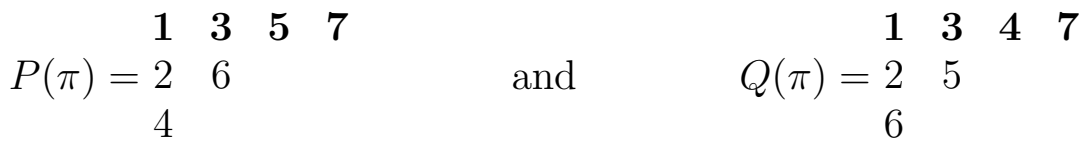

computed by Schensted Algorithm. It seems as if

$$
P_{1, j}=y_{L_{j}} \quad \text { and } \quad Q_{1, j}=x_{L_{j}}
$$

for all $j$.

In fact, even more is true. The boxes on line $L_{j}$ are precisely those elements passing through the $(1, j)$ cell during the construction of $P$, as the next result shows.

Lemma 2.3. Let the shadow diagram of $\pi=x_{1} x_{2} \cdots x_{n}$ be constructed as before. Suppose the vertical line $x=k$ intersects $i$ of the shadow lines. Let $y_{j}$ be the $y$-coordinate of the lowest point of the intersection with $L_{j}$. Then the first row of the $P_{k}=P\left(x_{1} \ldots x_{k}\right)$ is

$$
R_{1}=y_{1} y_{2} \cdots y_{i}
$$

Proof. : See [7]. 
EXAMPLE 2.4. Let $\pi=4236517$, then

$$
\begin{aligned}
& \begin{array}{l}
P_{0}=\emptyset \\
P_{1}=4
\end{array} P_{2}=\begin{array}{l}
2 \\
4
\end{array} \quad P_{3}=\begin{array}{ll}
2 & 3 \\
4 &
\end{array}
\end{aligned}
$$

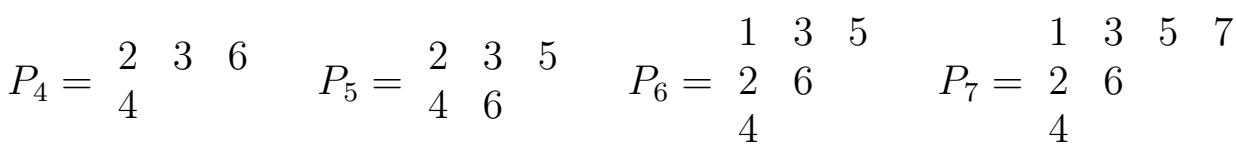

(Case 1) $k=3, P_{3}=P\left(x_{1} x_{2} x_{3}\right)=P(423)=\begin{array}{ll}2 & 3 \\ 4\end{array} \quad, \quad R_{1}=y_{1} y_{2}$

Then $x_{k+1}=x_{4}=6>3=y_{2}$ and so $\left(k+1, x_{k+1}\right)=(4,6)$ starts a new shadow line. Hence, $y_{3}=x_{4}=6$.

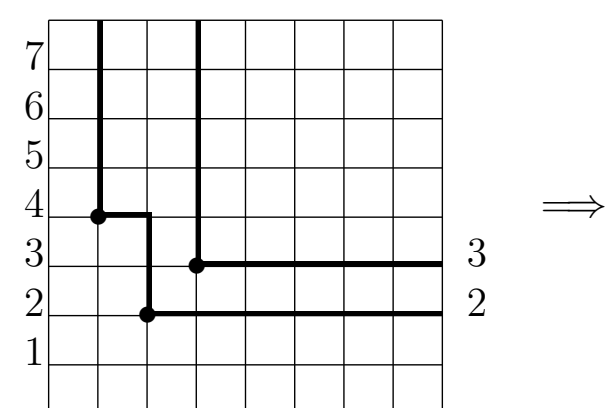

$\begin{array}{llllllll}0 & 1 & 2 & 3 & 4 & 5 & 6 & 7\end{array}$

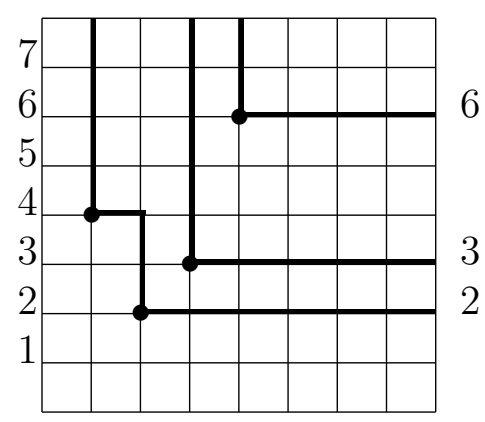

$\begin{array}{llllllll}0 & 1 & 2 & 3 & 4 & 5 & 6 & 7\end{array}$

(Case 2) $k=4, P_{4}=P(4236)=\begin{array}{lll}2 & 3 & 6 \\ 4\end{array} \quad, R_{1}=236=y_{1} y_{2} y_{3}$

Then $y_{1}<\cdots<y_{j-1}<x_{5}=5<y_{j}<\cdots<y_{3}=6$ and so $\left(k+1, x_{k+1}\right)=(5,5)$ is added to line $L_{j}$. Hence, $y_{j}^{\prime}=x_{k+1}=x_{5}=5$.

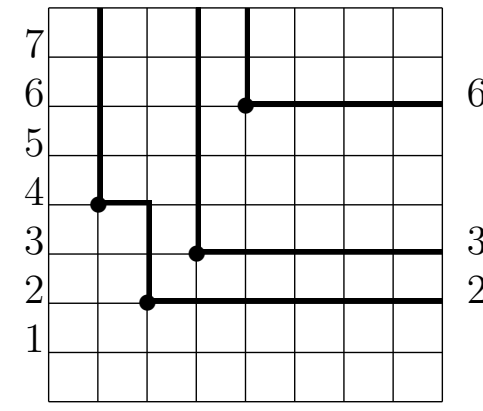

$\begin{array}{lllllllll}0 & 1 & 2 & 3 & 4 & 5 & 6 & 7\end{array}$

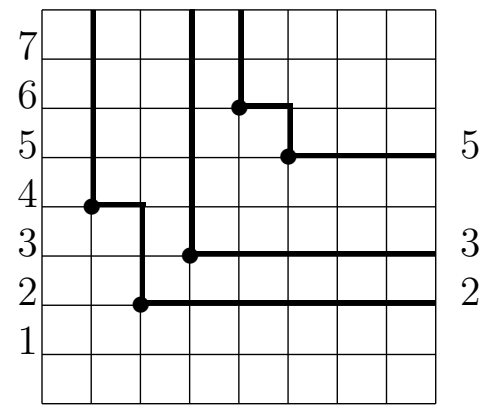

$\begin{array}{llllllll}0 & 1 & 2 & 3 & 4 & 5 & 6 & 7\end{array}$ 
It follows from the previous lemma that the shadow diagram of $\pi$ can be read left to right like a time-line recording the construction of $P(\pi)$. At the $k$-th stage, the line $x=k$ intersects one shadow line in a ray or line segment and all the rest in single points. In terms of the first row of $P_{k}$ : a ray corresponds to placing an element at the end, a line segment corresponds to displacing an element, and the points correspond to elements that are unchanged.

Corollary 2.5. If the permutation $\pi$ has Schensted tableaux $(P, Q)$ and shadow lines $L_{j}$, then, for all $j$,

$$
P_{1, j}=y_{L_{j}} \quad \text { and } \quad Q_{1, j}=x_{L_{j}} .
$$

Proof. See [7].

EXAMPLE 2.6. Let

$$
\pi=4236517 \in S_{7}
$$

Then the first, second and third rows come from the thickened and dashed lines, respectively, of the figure 5, 6 and 7 .

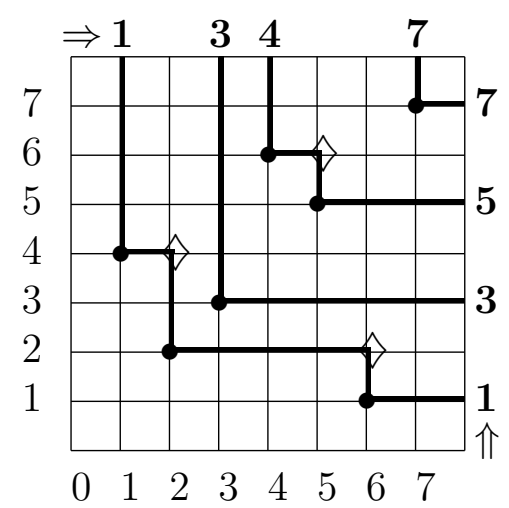

Figure 5. The first row of $P$ and $Q$.

The $\boldsymbol{i}$-th skeleton of $\pi \in S_{n}, \boldsymbol{\pi}^{(i)}$, is defined inductively by $\pi^{(1)}=\pi$ and

$$
\pi^{(i)}=\begin{array}{cccc}
k_{1} & k_{2} & \cdots & k_{m} \\
l_{1} & l_{2} & \cdots & l_{m}
\end{array}
$$

where $\left(k_{1}, l_{1}\right), \ldots,\left(k_{m}, l_{m}\right)$ are the NorthEast corners of the shadow diagram of $\pi^{(i-1)}$ listed in lexicographic order. The shadow lines for $\pi^{(i)}$ are denoted $\boldsymbol{L}_{\boldsymbol{j}}{ }^{(i)}$. 


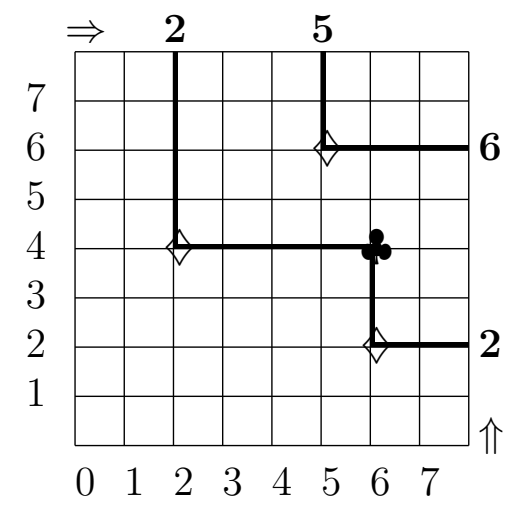

Figure 6 . The second row of $P$ and $Q$.

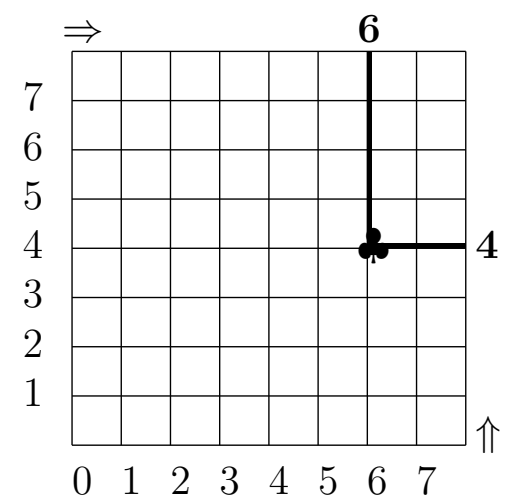

Figure 7 . The third row of $P$ and $Q$.

EXAmPLE 2.7. Let $\pi=4236517$ and

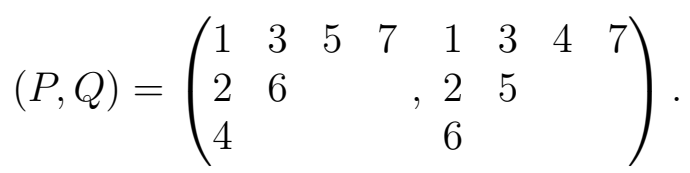

$\pi^{(2)}=\begin{array}{lll}2 & 5 & 6 \\ 4 & 6 & 2\end{array}$, where $\{4,6,2\}$ and $\{2,5,6\}$ are the remainder except for the first row of $P$ and $Q$, respectively.

$$
\begin{gathered}
P_{1, j}=y_{L_{j}^{(1)}}=y_{L_{1}^{(1)}} y_{L_{2}^{(1)}} y_{L_{3}^{(1)}} y_{L_{4}^{(1)}}=1357 \\
Q_{1, j}=x_{L_{j}^{(1)}}=x_{L_{1}^{(1)}} x_{L_{2}^{(1)}} x_{L_{3}^{(1)}} x_{L_{4}^{(1)}}=1347
\end{gathered}
$$




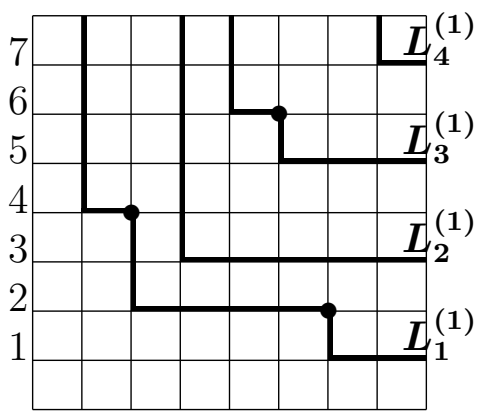

$\begin{array}{llllllll}0 & 1 & 2 & 3 & 4 & 5 & 6 & 7\end{array}$

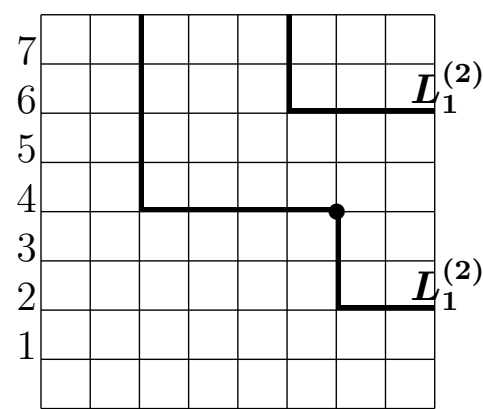

$\begin{array}{llllllll}0 & 1 & 2 & 3 & 4 & 5 & 6 & 7\end{array}$

$\pi^{(3)}=\begin{aligned} & 6 \\ & 4\end{aligned}$, where 4 and 6 are the remainder except for the first, second rows of $P$ and $Q$, respectively.

$$
P_{2, j}=y_{L_{j}^{(2)}}=y_{L_{1}^{(2)}} y_{L_{2}^{(2)}}=26 \quad, \quad Q_{2, j}=x_{L_{j}^{(2)}}=x_{L_{1}^{(2)}} x_{L_{2}^{(2)}}=25
$$

Continuing this processing $, P_{3, j}=y_{L_{1}^{(3)}}=4 \quad, \quad Q_{3, j}=x_{L_{1}^{(3)}}=6$.

Theorem 2.8. Suppose $\pi \rightarrow(P, Q)$. Then $\pi^{(i)}$ is a partial permutation such that

$$
\pi^{(i)} \rightarrow\left(P^{(i)}, Q^{(i)}\right)
$$

where $P^{(i)}$ (respectively, $Q^{(i)}$ ) consists of the rows $i$ and below of $P$ (respectively, $Q$ ). Furthermore,

$$
P_{i, j}=y_{L_{j}^{(i)}} \quad \text { and } \quad Q_{i, j}=x_{L_{j}^{(i)}}
$$

for all $i, j$.

Proof. See [7].

\section{Main results}

Given a permutation $\pi=x_{1} x_{2} \cdots x_{n-1} x_{n} \in S_{n}$, we define new permutations $\pi^{r}, \pi^{*}$ and $\pi^{\#}$ as follows.

$$
\begin{aligned}
\pi^{r} & =x_{n} x_{n-1} \cdots x_{2} x_{1} \\
\pi^{*} & =\left(n+1-x_{1}\right)\left(n+1-x_{2}\right) \cdots\left(n+1-x_{n-1}\right)\left(n+1-x_{n}\right) \\
\pi^{\#} & =\left(n+1-x_{n}\right)\left(n+1-x_{n-1}\right) \cdots\left(n+1-x_{2}\right)\left(n+1-x_{1}\right) .
\end{aligned}
$$

Note that $\left(\pi^{*}\right)^{r}=\pi^{\#}$. Until now we used Schützenberger's evacuation algorithm to compute $P$-tableaux and $Q$-tableaux of permutations 
$\pi^{r}, \pi^{*}$ and $\pi^{\#}$. See [7] for detail. In this section we describe the ways to find $P$-tableaux and $Q$-tableaux of permutations $\pi^{r}, \pi^{*}$ and $\pi^{\#}$ directly from Viennot's geometric interpretation for Schensted algorithm.

Given a permutation $\pi \in S_{n}$, let $\pi \stackrel{[\mathrm{S}]}{\longleftrightarrow}(P, Q)$. Then we can find $P$-tableaux and $Q$-tableaux of permutations $\pi^{r}, \pi^{*}$ and $\pi^{\#}$ as the following propositions.

Proposition 3.1. Let $\pi^{*} \stackrel{[\mathrm{S}]}{\longleftrightarrow}\left(P^{*}, Q^{*}\right)$. Then $\left(P^{*}, Q^{*}\right)$ can be obtained as follows:

1. Imagine a light shining from $(0, n+1)$ and get a new shadow diagram of $\pi$.

2. Change any coordinate $y_{L_{i}}$ to $(n+1)-y_{L_{i}}$.

3. $P$-tableaux and $Q$-tableaux are obtained similarly as if we read the original shadow diagram of $\pi$.

EXAmPle 3.2. Let $\pi=4236517$. Then,

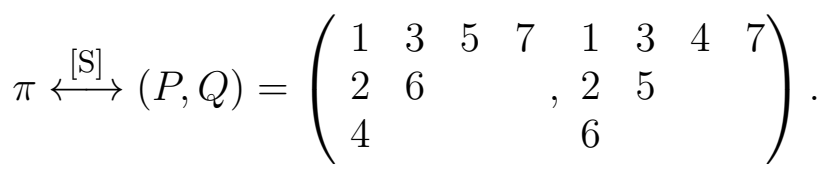

By the reading coordinate steps of Figure 8 and 9, we obtain that

$$
\left(P^{*}, Q^{*}\right)=\left(\begin{array}{llllll}
1 & 3 & 7 & 1 & 2 & 6 \\
2 & 5 & & 3 & 5 & \\
4 & & & 4 & & \\
6 & & & 7 & &
\end{array}\right)
$$

Note that $P^{*}=(\mathrm{ev}(P))^{t}$ and $Q^{*}=Q^{t}$.

Proposition 3.3. Let $\pi^{r} \stackrel{[\mathrm{S}]}{\longleftrightarrow}\left(P^{r}, Q^{r}\right)$. Then $\left(P^{r}, Q^{r}\right)$ can be obtained as follows:

1. Imagine a light shining from $(n+1,0)$ and get a new shadow diagram of $\pi$.

2. Change any coordinate $x_{L_{i}}$ to $(n+1)-x_{L_{i}}$.

3. P-tableaux and $Q$-tableaux are obtained similarly as if we read the original shadow diagram of $\pi$. 
$\Downarrow$

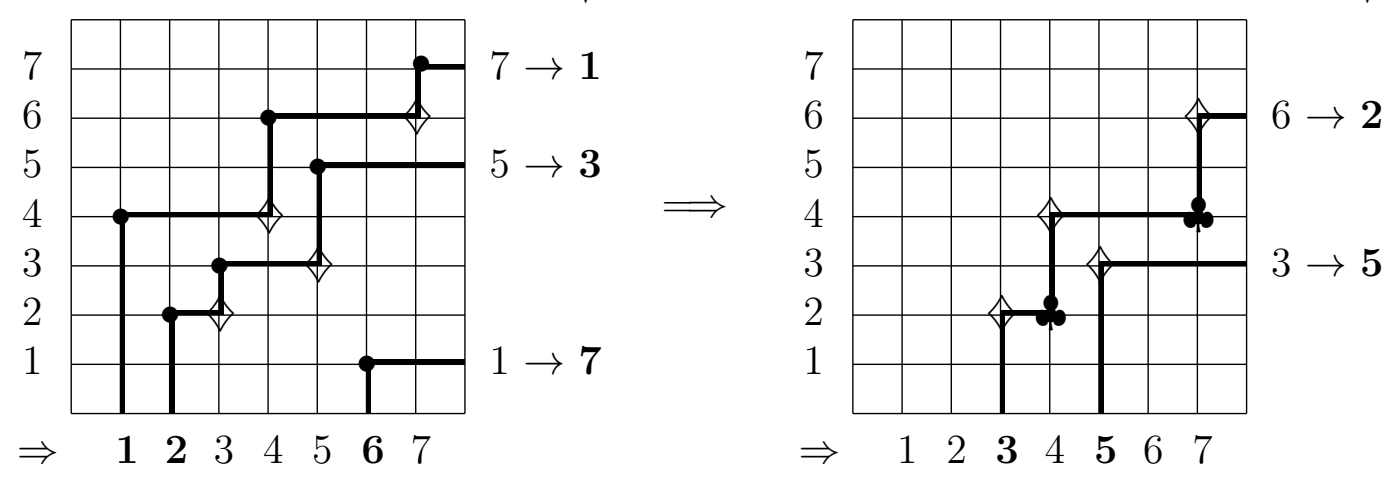

Figure 8. First and Second rows for $\pi^{*}$

$\Downarrow$
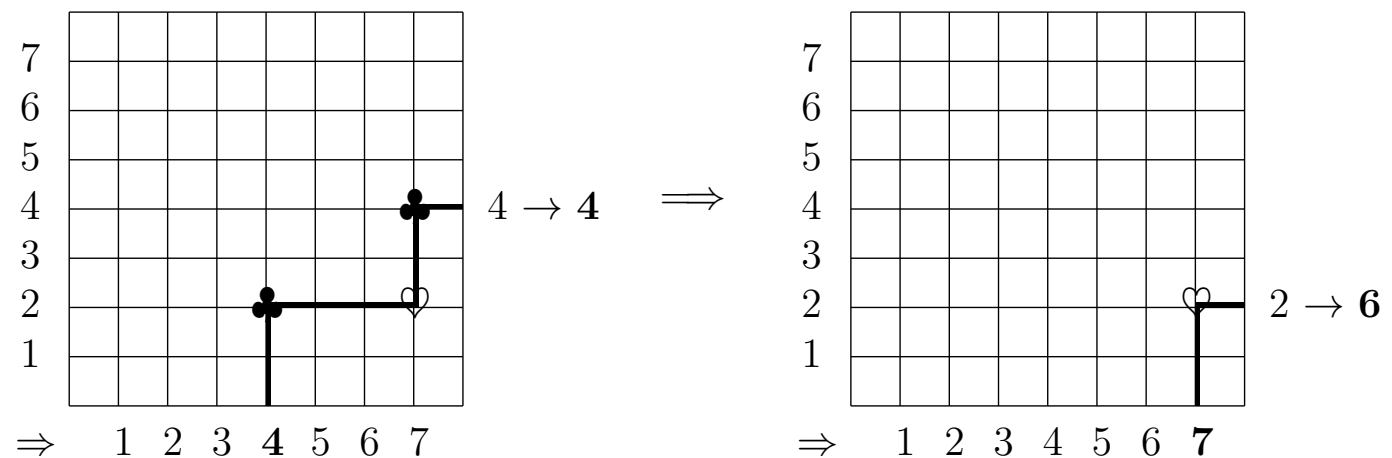

FiguRE 9. Third and Fourth rows for $\pi^{*}$

EXAMPLE 3.4. Let $\pi=4236517$. By the reading coordinate steps of Figure 10 and 11, we obtain that

$$
\left(P^{r}, Q^{r}\right)=\left(\begin{array}{llllll}
1 & 2 & 4 & 1 & 3 & 4 \\
3 & 6 & & 2 & 7 & \\
5 & & & 5 & & \\
7 & & & 6 & &
\end{array}\right)
$$

Note that $P^{r}=P^{t}$ and $Q^{r}=(\mathrm{ev}(Q))^{t}$.

Proposition 3.5. Let $\pi^{\sharp} \stackrel{[\mathrm{S}]}{\longleftrightarrow}\left(P^{\sharp}, Q^{\sharp}\right)$. Then $\left(P^{\sharp}, Q^{\sharp}\right)$ can be obtained as follows:

1. Imagine a light shining from $(n+1, n+1)$ and get a new shadow diagram of $\pi$. 
Jaejin Lee
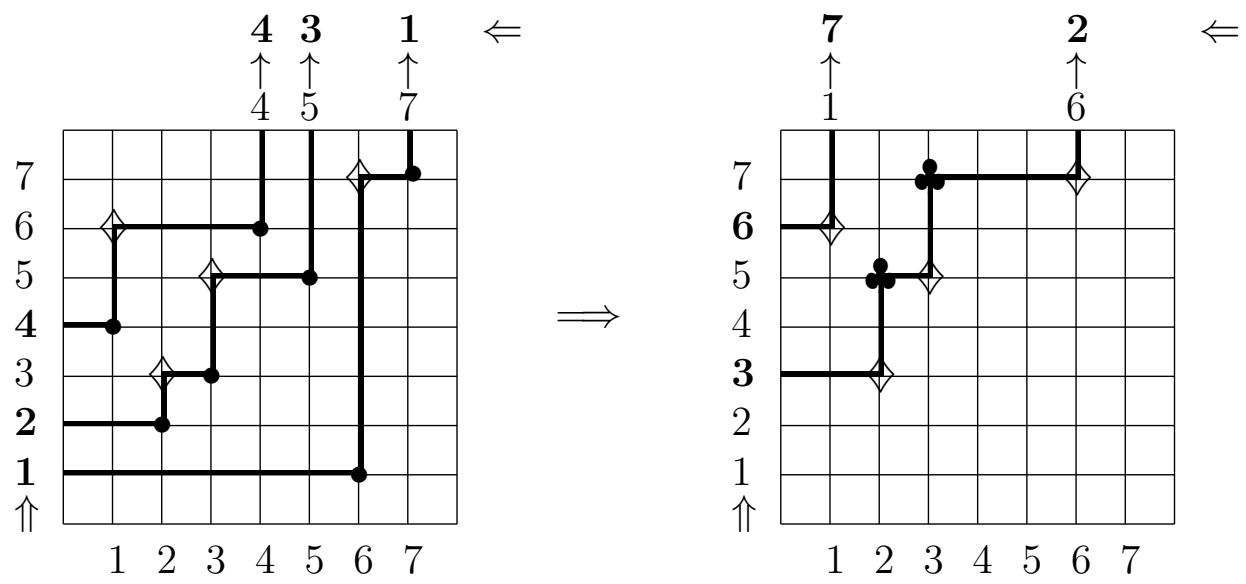

FiguRE 10. First and Second rows for $\pi^{r}$
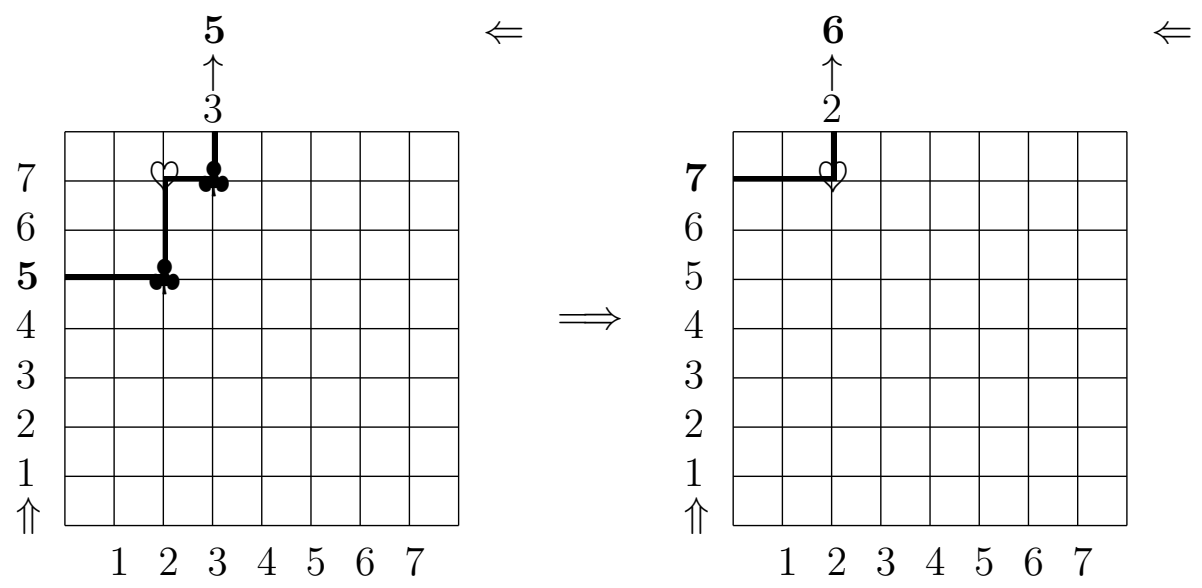

FiguRE 11. Third and Fourth rows for $\pi^{r}$

2. Change any coordinate $x_{L_{j}}$ and $y_{L_{i}}$ to $(n+1)-x_{L_{j}}$ and $(n+1)-y_{L_{i}}$, respectively.

3. $P$-tableaux and $Q$-tableaux are obtained similarly as if we read the original shadow diagram of $\pi$.

EXAMPLE 3.6. Let $\pi=4236517$. By the reading coordinate steps of Figure 12 and 13, we obtain that

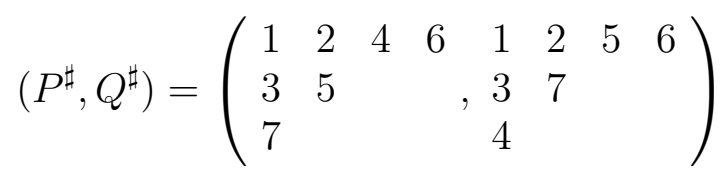


Note that $P^{\sharp}=\mathrm{ev}(P)$ and $Q^{\sharp}=\mathrm{ev}(Q)$.

$\Downarrow$

$\Downarrow$

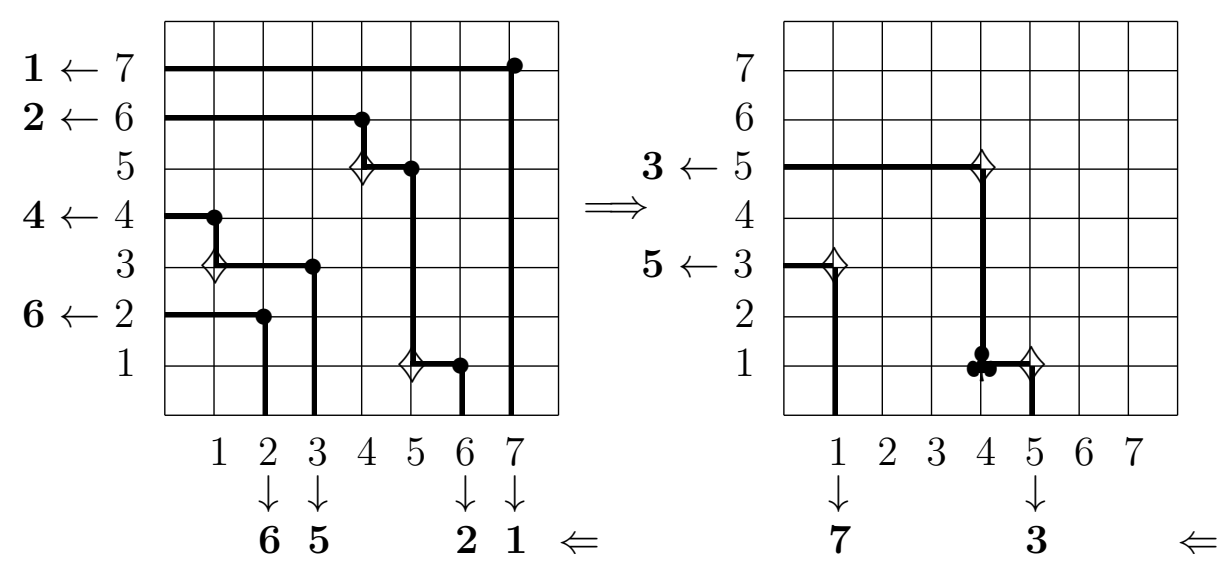

Figure 12. First and Second rows for $\pi^{\sharp}$

$\Downarrow$

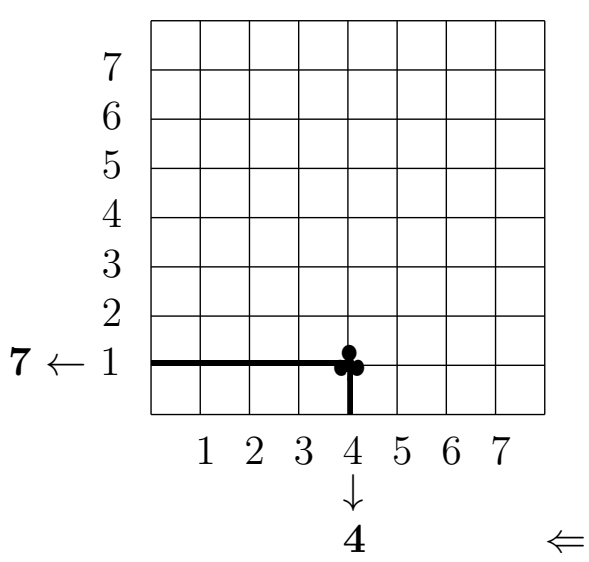

FiguRE 13. Third row for $\pi^{\sharp}$

\section{References}

[1] A. Berele, A Schensted-type correspondence for the symplectic group, J. Combin. Theory Ser. A 43 (1986), 320-328. 
[2] C. Greene, An extension of Schensted's theorem, Adv. in Math., 14 (1974), $254-265$.

[3] J. J. Lee, A Schensted algorithm for shifted rim hook tableaux, Korean J. Math., 31 (1994), 179-203.

[4] D. E. Knuth, Permutations, matrices and generalized Young tableaux, Pacific J. Math., 34 (1970), 709-727.

[5] G. de B. Robinson, On the representations of the symmetric group , Amer. J. Math., 60, (1938), 745-760.

[6] B. E. Sagan, Shifted tableaux, Schur $Q$-functions and a conjecture of R. Stanley, J. Combin. Theory Ser. A 45 (1987), 62-103.

[7] B. E. Sagan, The symmetric group, Springer-Verlag, New York, 2000.

[8] B. E. Sagan and R. P. Stanley, Robinson-Schensted algorithms for skew tableaux, J. Combin. Theory Ser. A, 55 (1990), 161-193.

[9] C. Schensted, Longest increasing and decreasing subsequences, Canad. J. Math., 13 (1961), 179-191.

[10] D. W. Stanton and D. E. White, A Schensted algorithm for rim hook tableaux, J. Combin. Theory Ser. A 40 (1985), 211-247.

[11] M. P. Schützenberger, Quelques remarques sur une construction de Schensted, Math., Scand. 12 (1963), 117-128.

[12] G. Viennot, Une forme géométrique de la correspondance de RobinsonSchensted, in Combiatoire et Représentation du Groupe Symétrique, D. Foata ed., Lecture Notes in Math., Vol. 579, Springer-Verlag, New York, NY, 1977, $29-58$.

Department of Mathematics

Hallym University

Chunchon, Korea 200-702

E-mail: jjlee@hallym.ac.kr 